\title{
Size determination of the Centaur Chariklo from millimeter-wavelength bolometer observations
}

\author{
W. J. Altenhoff, K. M. Menten, and F. Bertoldi \\ Max-Planck-Institut für Radioastronomie, Auf dem Hügel 69, 53121 Bonn, Germany \\ Received 27 November 2000 / Accepted 15 December 2000

\begin{abstract}
Using the Max-Planck Millimeter Bolometer Array (MAMBO) at the IRAM 30 m telescope we detected emission at $250 \mathrm{GHz}$ from the Centaur Chariklo (1997 CU26). The observed continuum flux density implies a photometric diameter of $273 \mathrm{~km}$. The resulting geometric albedo is 0.055 , somewhat higher than expected from a comparison with most of the other few Centaurs and cometary nuclei for which such data are available.
\end{abstract}

Key words. comets: general - minor planets, asteroids - radio continuum: solar system

\section{Introduction}

Within the last decade, our view of the outer solar system has been revolutionized by the discovery of a large number of "trans-Neptunian" objects, which appear to be remnant planetesimals from the formation of the solar system (Jewitt 1999). These so-called (Edgeworth-) Kuiper Belt objects (EKOs, KBOs, or TNOs) have orbits in the range from 30 to $50 \mathrm{AU}$ (see Jewitt \& Luu 2000 for a review). Near the Kuiper Belt, two other populations of asteroids were detected recently, the Centaurs (named by Kowal 1989 after his detection of Chiron), which have perihelia between Jupiter and Neptune, and the scattered disk objects (SDO, or SKDO), having perihelia near $35 \mathrm{AU}$, semimajor axes near $85 \mathrm{AU}$, high eccentricities, and intermediate inclinations (Chadwick et al. 2000).

Systematic surveys for these objects allow estimates of their total populations. Based on their survey, Jewitt et al. (1996) deduce a total number of 70000 EKOs with diameters, $d \geq 100 \mathrm{~km}$ and of 2600 Centaurs with $d \geq 70 \mathrm{~km}$. Chadwick et al. (2000) estimate that there are 31000 SDOs with $d \geq 100 \mathrm{~km}$. According to Parker (2000), presently 330 EKOs and 55 Centaurs (including 5 SDOs) are known with their orbital elements; during the last few years the numbers of catalogued EKOs and Centaurs have grown exponentially, more than doubling each year. The obvious reasons why these distant asteroids have been detected only recently are their "small" sizes and low albedos, resulting in faint optical emission $\left(m_{V} \sim 19-24\right)$ and very low infrared (IR) and radio flux densities. The resulting lack of precise thermal

Send offprint requests to: W. J. Altenhoff, e-mail: waltenhoff@mpifr-bonn.mpg.de (i.e. infrared-, (sub)millimeter-, or radio-wavelength) measurements in turn precludes accurate albedo and size determinations.

These distant asteroids (and comets) are of great interest because they are likely to consist of pristine and mostly unaltered matter from the solar nebula. Their nearly black surface is thought to be the result of cosmic particle bombardment (e.g., Johnson et al. 1987). The KBOs move in a dynamically stable region, while the Centaurs orbit in a dynamically unstable range between the major gas planets, which limits their dynamical life times to about $10^{6}$ years. The unexpectedly large population of Centaurs can only be maintained by continuous replenishment from the reservoir of EKOs (Stern \& Campins 1996). Similarly, the SDOs are thought to be ejected from the Kuiper Belt by Neptune scattering (Chadwick et al. 2000).

Circumstantial evidence for a Kuiper belt origin of the Centaurs comes from the facts that both, EKOs and Centaurs, have similar reflectance spectra, cover probably a similar size range, and probably have similar albedos (see below). Numerical orbit integrations by Levison \& Duncan (1997) indicate that at the end of its lifetime a Neptune encountering body such as a Centaur may become a Jupiter family short period "comet". Indeed, attempts to subdivide these distant icy bodies into asteroids on the one hand and comets one the other seem artificial. For example, Chiron shows many of the characteristics of a comet and yet is the prototypical Centaur, while the bona fide comets P/Oterna and P/Schwassmann-Wachmann I are listed by Jewitt \& Kalas (1998) and Jewitt \& Luu (2000) as Centaurs.

Optical wavelength reflection spectra are of fundamental importance for understanding the nature of these 
bodies. From their observations of five KBOs, Jewitt \& Luu (1998) found a linear relationship between the $V-J$ color index and the absolute red magnitude $M_{R}$. Tegler \& Romanishin (1998) observed a few KBOs and Centaurs, finding a bimodal distribution in the $B-V, V-R$ color diagram for both types of asteroids. Davies et al. (2000) obtained photometry for a larger sample of KBOs. While they confirm previous measurements for those objects of their sample that had been studied before, their results in total make the existence a color-distance correlation and a bimodal distribution of KBOs appear less convincing.

Using optical data alone, the sizes of these distant bodies can only be estimated from the observed optical magnitude by assuming a geometric ( $V$-band) albedo $p_{V}$. Jewitt \& Luu (1995) used for their population statistics a "comet-like geometric albedo of 0.04 ", but it is still unknown how good an assumption this is, considering that the Pluto-Charon binary, frequently classified as a KBO, has a geometric albedo around 0.60 .

Given these uncertainties, the derived diameters can be wrong by more than a factor of 2 . This is an unfortunate situation, since an accurate size determination is an indispensable prerequisite for any physical interpretation, in particular for deriving surface properties and the mass of a single object and, thus, the total mass contained in all the distant asteroids. There is obviously an urgent need for good size and albedo determinations.

Earlier observations with the IRAM $30 \mathrm{~m}$ telescope have shown that precise mean size determinations of asteroids can be made using careful photometry near a frequency of $250 \mathrm{GHz}(1.2 \mathrm{~mm}$ wavelength; Altenhoff et al. 1994; Altenhoff \& Stumpff 1995). Altenhoff et al. (1996) showed that the quality of a mm-wavelength photometric size determination can match that of occultation measurements, if the mm observations cover the rotation period of the asteroid.

Given their large distances and small diameters EKOs are expected to be very faint at millimeter wavelengths. Expected $250 \mathrm{GHz}$ flux densities for the largest of these objects $(d \approx 600 \mathrm{~km})$ at a typical distance of $40 \mathrm{AU}$ are around $0.5 \mathrm{mJy}$. Given this, their detection would require prohibitive amounts of observing time even with the largest millimeter-wavelength telescopes equipped with sensitive bolometer detectors. However, due to their proximity, the flux densities of some Centaurs should be higher and observing them would yield results that are relevant to KBOs as well. For example, the signal of Chariklo, formerly known as 1997 CU26, is expected to be five times stronger than the above value and thereby detectable. This Centaur was detected with the Spacewatch telescope in 1997 (Scotti 1997). It has an absolute magnitude, $H$ (i.e. visual magnitude, reduced to $1 \mathrm{AU}$ geocentric and heliocentric distance and phase angle 0; see, e.g., Bowell et al. 1989) of 6.4 , corresponding to an estimated diameter of $353 \mathrm{~km}$, when assuming an albedo of 0.04 . It is the largest known Centaur and has a very dark surface, supposedly comparable to that of Pholus (Mueller et al. 1992).
Mid-infrared photometry at $20 \mu \mathrm{m}$ by Jewitt \& Kalas (1998) resulted in an improved diameter of $302 \mathrm{~km}$. This value depends on assumptions of the asteroidal model, the IR emissivity, IR phase, the "beaming parameter", etc., which are not all accurately known. This can introduce systematic uncertainties in the size estimate that are bigger than the quoted error of $30 \mathrm{~km}$. In contrast, we know from experience that at $250 \mathrm{GHz}$ the emissivity, $e$, and the beaming parameter are close to unity and that the phase effect is negligible (for discussions of these quantities see Spencer et al. 1989; Altenhoff et al. 1994; Jewitt \& Kalas 1998). Thus (sub)millimeter observations in principle are capable of yielding more accurate diameter estimates than IR data. In the following we describe our $250 \mathrm{GHz}$ observations of Chariklo.

\section{Observations}

Our observations were made with the IRAM $30 \mathrm{~m}$ telescope on Pico Veleta, Spain, using a 37 channel version of the Max-Planck Millimeter Bolometer (MAMBO), built at the Max-Planck-Institut für Radioastronomie (Kreysa et al. 1998). This is a hexagonally close packed, diffraction limited bolometer array operating at $0.3 \mathrm{~K}$. A common filter defines a bandpass centered at $250 \mathrm{GHz}$, if folded with black body spectra corresponding to minor planets. The beamwidth is $11^{\prime \prime}$ (FWHM). Observing modes included drift scans for pointing and calibration measurements and an ON-OFF technique described by Bertoldi et al. (2000), which provides high (sub-mJy) sensitivity by employing sky noise subtraction.

The flux density scale was derived from observations of asteroid 1 Ceres (see Altenhoff et al. 1996) and the planets Mars and Uranus from which a calibration factor of 12500 counts per Jy was derived.

The calculation of the ephemerides for the minor planets 1 Ceres and 10199 Chariklo was based on the orbital elements, published by Shor (1999) and Marsden \& Williams (1999), respectively, adjusting the osculating epoch to the date of observations by taking into account the perturbations by the major planets. Since the orbital elements for Chariklo were determined from 344 observations covering 11(!) years (including prediscovery data), the resulting position accuracy is $\sim 0.5^{\prime \prime}$ and thus easily sufficient for our observations.

Typical rotation periods of asteroids and comets are between 5 and 15 hours (see, e.g., light curve parameters given by Shor 1999). Since Chariklo's rotation period is unknown, we observed it during six short periods on different days in order to cover different intervals of a possibly existing "light curve". Averaging the data from these individual observations will yield a mean diameter. Given that the expected signal was only $\approx 2.5$ mJy precluded an actual measurement of light variations with plausible amplitudes or obtaining constraints on the three-dimensional shape of this Centaur.

We observed Chariklo between 1999 December 9 and 2000 February 29 at night time under good weather 
conditions. Its mean geocentric distance, $\Delta$, was $12.62 \mathrm{AU}$ and its mean heliocentric distance, $r, 13.35$ AU. The data were reduced using an improved version of the MOPSI package developed by R. Zylka, allowing for effective sky noise subtraction and calibration, resulting in a higher sensitivity than attainable with previous data reduction procedures.

Our observations yield a mean $250 \mathrm{GHz}$ flux density of $S_{\nu}=2.08 \pm 0.30 \mathrm{mJy}$ after a total integration time of 2.4 hours; Fig. 1 shows the signals of the bolometer channel centered on Chariklo and the other bolometer channel (showing noise) as a function of the integration time.

\section{Results and discussion}

Assuming a diameter of $302 \mathrm{~km}$ and a geometric albedo of 0.045 , as derived by Jewitt \& Kalas (1998), we predict a flux density of $2.54 \mathrm{mJy}$ using the equations given by Altenhoff et al. (1994). Reversing the argument, we can use above geometric albedo (which we assume to be equal to the Bond albedo $A$; see Jewitt \& Kalas 1998) to calculate Chariklo's equilibrium temperature $T_{\text {eq }}$ from $T_{\text {eq }}=f(1-A)^{0.25} r^{-0.5}$, where $f=277 \mathrm{~K}$ for "fast rotation", a condition met by almost all asteroids, and $r$ is measured in AU. The diameter, $d$, is determined from $S_{\nu}=\frac{\pi d^{2}}{4 \Delta} e B_{\nu}\left(T_{\mathrm{B}}\right)$, where $e=1$ (see above), $T_{\mathrm{B}}$ is the brightness temperature above the cosmic microwave background $\left(T_{\mathrm{B}}=T_{\mathrm{eq}}-2.73 \mathrm{~K}\right)$, and $B_{\nu}(T)$ is the Planck function. With $S_{\nu}=2.08 \pm 0.30 \mathrm{mJy}$ we obtain $d=273 \pm 19 \mathrm{~km}$. Plugging this newly derived diameter into eq. (1) of Jewitt \& Kalas (1998), yields a new value of $0.055 \pm 0.008$ for the geometric albedo. Our assumption of fast rotation should - at millimeter wavelengths - apply for all rotation periods under 50 hours. It appears to be supported by the results of Peixinho et al. (2000), who tried to determine Chariklo's optical light curve, finding a rotation period of either 15 or $34 \mathrm{~h}$.

Assuming no rotation or, equivalently, that the asteroidal rotation axis is pointing toward the observer, one calculates for the sun-lit surface an equilibrium temperature that is $19 \%$ higher than the fast rotation value, resulting in a $9 \%$ smaller derived diameter. Applying this to our numbers yields $d=250 \mathrm{~km}$ and $p_{V}=0.065$ for Chariklo. Since the surface depth probed by thermal emission is approximately proportional to the wavelength, the time scale discriminating between the fast or slow rotation scenarios is also wavelength dependent. Thus, while our adoption of fast rotation appears to be appropriate for $\mathrm{mm}$ wavelengths, the no rotation assumption of Jewitt \& Kalas (1998) may be applicable to their $20 \mu \mathrm{m}$ data.

Above albedo values for Chariklo are similar to 0.04, the value often assumed for EKOs and related objects. Table 1 presents a critically selected collection of the few meaningful diameter and albedo determinations of Centaurs and cometary nuclei that are available in the literature. As discussed above, Centaurs and the short period Jupiter comets are likely to originate from the Kuiper
Table 1. Diameters and albedos of Centaurs and cometary nuclei

\begin{tabular}{lcllc}
\hline Object & $\begin{array}{c}\text { Band/ } \\
\text { Method }\end{array}$ & $\begin{array}{l}\text { Diameter } \\
{[\mathrm{km}]}\end{array}$ & $\begin{array}{l}p_{V}^{\mathrm{a}} \\
(\%)\end{array}$ & Ref. \\
\hline Chariklo & $20 \mu \mathrm{m}$ & 302 & $4.5 \pm 1.0$ & 1 \\
& $1.2 \mathrm{~mm}$ & 275 & $5.5 \pm 0.5$ & 2 \\
Chiron & $10 / 20 \mu \mathrm{m}$ & 180 & $14_{-3}^{+6}$ & 3 \\
& $1.2 \mathrm{~mm}$ & 168 & $13_{-3}^{+4}$ & 4 \\
& occult. $^{\mathrm{b}}$ & 179.2 & $14.8 \pm 2.2$ & 5 \\
Pholus & $20 \mu \mathrm{m}$ & 189 & $4.4 \pm 1.3$ & 6 \\
P/Halley & Giotto $^{\mathrm{c}}$ & 10 & $3.9 \pm 0.3$ & 7,8 \\
P/Arend-Rigaux & $5-20 \mu \mathrm{m}$ & 10 & $2.8 \pm 0.5$ & 9 \\
P/Neujmin 1 & $10 / 20 \mu \mathrm{m}$ & 20 & $2.5 \pm 0.5$ & 10 \\
P/Tempel 2 & $20 \mu \mathrm{m}$ & 11.8 & $2.2_{-0.6}^{+0.4}$ & 11 \\
\hline
\end{tabular}

a Visual geometric or albedo.

b Diameter determination using stellar occultation.

c Diameter determination using data from the Giotto spacecraft.

References: (1) Jewitt \& Kalas (1998), (2) this paper, (3) Campins et al. (1994), (4) Altenhoff \& Stumpff (1995), (5) Bus et al. (1996), (6) Davies et al. (1993), (7) Jewitt et al. (1982), albedo derived from diameter in ref. 8, (8) Keller et al. (1987), (9) Millis et al. (1988), (10) Campins et al. (1987), (11) A'Hearn et al. (1989).

belt. Our millimeter data for Chariklo, confirm the earlier IR data for this object.

The derived albedo values vary from 0.02 to possibly 0.14 , but the median value is still near 0.04 . We note Chiron's high albedo, meticulously derived by Campins et al. (1994), who subtracted the coma contribution. Whether this high geometric albedo is unique, is presently difficult to answer. The Pluto-Charon binary with its albedo of $\sim 0.6$ (Marcialis et al. 1992) may have a different structure than typical EKOs. We further note that $\mathrm{C} /$ Hale-Bopp may have a high albedo around 0.3. This estimate is based on this comet's unexpectedly small nuclear diameter indicated by the millimeter observations of Altenhoff et al. (1999) and its absolute magnitude, $H$, of $\approx 8.7$, which is derived from the pre-discovery observations of McNaught (1995). However, Hale-Bopp's albedo determinations have to be viewed with caution since this object showed cometary activity already at a distance $\Delta \geq 13$ AU. It is conceivable that the higher albedos of active Centaurs/comets are caused by the partial loss of their dark surface covers.

While the similar albedos of Centaurs and cometary nuclei may indicate a relation between both classes of objects, these groups have quite different reflection spectra and size distributions. The latter point is hinted at by the data compiled in Table 1, although the statistics are poor. Moreover, according to Luu \& Jewitt (1996) most of the Centaurs differ from cometary nuclei and Trojan asteroids by their extreme red color and IR absorption lines. Recently, Tegler \& Romanishin (2000) reported that most of the observed KBOs show extremely red colors as well. 


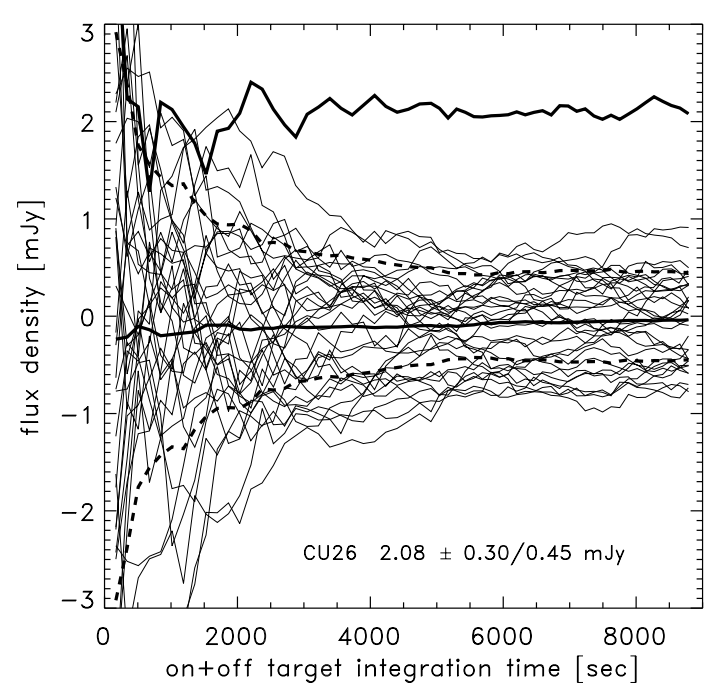

Fig. 1. Observed signal of Centaur Chariklo. Plotted is the integrated signal for all bolometer channels as function of integration time. Channel 1 (on source) is plotted as heavy line, showing a signal of $2.08 \mathrm{mJy}$. The dashed lines indicate the rms dispersion of all off-source channels, which is $0.45 \mathrm{mJy}$

Thus there seem to be marked differences between KBOs and Centaurs on the one side and cometary nuclei and Trojans on the other. Nevertheless, the above does not necessarily exclude the possibility that the Jupiter family comets originate in the Kuiper belt. However, if that scenario were true, the data seem to imply that the sizes and surfaces of these objects are substantially altered during their way to the Sun.

\section{Conclusions and outlook}

Our millimeter wavelength detection of the Centaur Chariklo yields an unbiased size determination and an albedo estimate for this object. Since Centaurs are very likely to be Edgeworth-Kuiper-Objects that have moved inwards from the Kuiper Belt, our results may also have implications for bona fide EKOs. At distances around $40 \mathrm{AU}$, these will be very difficult to detect with today's millimeter-wavelength instrumentation. However, with next generation facilities such as the Atacama Large Millimeter Array (ALMA) it will not only be possible to detect hundreds of EKOs. ALMA will also allow interferometric, direct size measurements of such objects (see, e.g., Menten 2000).

Acknowledgements. We would like to thank Ernst Kreysa and the MPIfR bolometer group, Robert Zylka for providing an improved version of his MOPSI data reduction package, Clemens Thum for flexibly scheduling this project as required by its need for optimum observing conditions, and the IRAM staff at the $30 \mathrm{~m}$ telescope for their able technical support.

\section{References}

A'Hearn, M. F., Campins, H., Schleicher, D. G., \& Millis, R. L. 1989, ApJ, 347, 1155
Altenhoff, W. J., Baars, J. W. M., Schraml, J. B., Stumpff, P., \& von Kap-herr, A. 1996, A\&A, 309, 953

Altenhoff, W. J., Johnston, K. J., Stumpff, P., \& Webster, W. J. 1994, A\&A, 287, 641

Altenhoff, W. J., \& Stumpff, P. 1995, A\&A, 293, L41

Altenhoff, W. J., et al. 1999, A\&A, 348, 1020

Bertoldi, F., et al. 2000, A\&A, 360, 92

Bowell, E., Hapke, B., Domingue, D., et al. 1989, in Asteroids II, ed. R. P. Binzel, T. Gehrels, \& M. S. Shapley (Univ. Arizona Press, Tucson), 524

Bus, S. J., et al. 1996, Icarus, 123, 478

Campins, H., A'Hearn, M. F., \& McFadden, L.-A. 1987, ApJ, 316,847

Campins, H., Telesco, C. M., Osip, D. J., et al. 1994, AJ, 108, 2318

Chadwick, A., Trujillo, C., Jewitt, D. C., \& Luu, J. X. 2000, ApJ, 529, L103

Davies, J., Spencer, J., Sykes, M., Tholen, D., \& Green, S. 1993, IAU Circ., 5698

Davies, J. K., et al. 2000, Icarus, 146, 253

Jewitt, D. 1999, Annu. Rev. Earth Planet. Sci., 27, 287

Jewitt, D., \& Kalas, P. 1998, ApJ, 499, L103

Jewitt, D., \& Luu, J. X. 1995, AJ, 109, 1867

Jewitt, D., \& Luu, J. 1998, AJ, 115, 1667

Jewitt, D., Luu, J., \& Chen, J. 1996, AJ, 112, 1225

Jewitt, D. C., Luu, J. X. 2000, in Protostars and Planets IV, ed. V. Mannings, A. P. Boss, S. S. Russell (Univ. Arizona Press, Tucson), 1201

Jewitt, D. C., et al. 1982, IAU Circ., 3737

Johnson, R. E., Cooper, J. F., Lanzerotti, L. J., \& Strazzulla, G. 1987, A\&A, 187, 889

Keller, H. U., Delamare, W. A., Huebner, W. F., et al. 1987, A\&A, 187, 807

Kowal, C. T. 1989, Icarus, 77, 118

Kreysa, E., et al. 1998, in Advanced Technology MMW, Radio, and Terahertz Telescopes, ed. T. G. Phillips, Proc. SPIE 3357,319

Levison, H. F., \& Duncan, M. J. 1997, Icarus, 127, 13

Luu, J., \& Jewitt, D. 1996, AJ, 112, 2310

Luu, J. 1997, Nature, 387, 573

Marcialis, R. L., Lebofsky, L. A., DiSanti, M. A., et al. 1992, AJ, 103, 1389

Marsden, B. G., \& Williams, G. V. 1999, Minor Planet Circular No. 33905

McNaught, R. H. 1995, IAU Circ., 6198

Menten, K. M. 2000, in ESO Astrophysics Symposia, From Extrasolar Planets to Cosmology: The VLT Opening Symposium, ed. J. Bergeron, \& A. Renzini (Springer, Berlin), 78

Millis, R. L., A'Hearn, M. F., \& Campins, H. 1988, ApJ, 324, 1194

Parker, J. W. 2000, The Kuiper Belt Electronic Newsletter No. 12

Peixinho, N., Lacerda, P., Roos-Serote, M., Ortiz, J., \& Doressoundiram, A. 2000, BAAS, 32, 961

Scotti, J. V. 1997, Minor Planet Circular 1997-D11

Shor, V. A. (ed.) 1999, Ephemerides of Minor Planets, St. Petersburg

Spencer, J. R., Lebofsky, L., \& Sykes, M. V. 1989, Icarus, 78, 337

Stern, A., \& Campins, H. 1996, Nature, 382, 507

Tegler, S. C., \& Romanishin, W. 1998, Nature, 392, 49

Tegler, S. C., \& Romanishin, W. 2000, Nature, 407, 979 